\title{
Plant Age and Isolate Source Affect Expression of Downy Mildew Resistance in Broccoli
}

\section{M.H. Dickson and R. Petzoldt \\ New York State Agricultural Experiment Station, Cornell University, Geneva, NY 14456 \\ Additional index words. Peronospora parasitica, disease resistance, Brassica oleracea Botrytis Group}

\begin{abstract}
Resistance to downy mildew [Peronospora parasitica (pers.) ex. Fr.] in broccoli (Brassica oleracea L. Italica Group) depends on plant age. Seedling resistance seems to be independent of mature-plant resistance, where a mature plant is defined as having eight or more leaves. Our results suggest that, by using mature-plant resistance, an almost continuous variation instable levels of mature-plant resistance can be developed. Similarities in the response of mature plants of various lines to isolates from California, Washington, New York, and South Carolina indicated that the predominant race was the same at all locations. Correlations between resistant and susceptible responses to isolates from California, Washington, New York, and South Carolina varied from $r=0.48$ to 0.74 depending on isolate source. The results indicated that selecting for high levels of resistance in mature plants at one location should result in good resistance elsewhere in the United States. Selecting immature plants (three to six leaves) may provide less reliable results due to the transitional status of the plant; i.e., between seedling and mature plant.
\end{abstract}

Brassica oleracea downy mildew (DM), caused by Peronospora parasitica, is a worldwide problem, but is more severe in cool climates with frequent dew formation at night. High disease levels can reduce yield and market quality. Severe disease on broccoli and cauliflower (B. oleracea Botrytis Group) heads and crops such as collards $(B$. oleracea Acephala Group), of which the large leaves are consumed, can make the crop unmarketable. Disease during seed production can reduce yields and seed quality and may even kill the crop before seeds mature.

Natti et al. (1967) identified two DM races on B. oleracea and reported that resistance to these races was due to independent dominant genes. A third race also was found (unpublished data) that occurred on senescing leaves late in the season. Although the segregation for both race 1 and 2 fit a 3:1 ratio, they reported that there were plants with lower degrees of resistance, a result indicating the presence of other genetic factors.

For many years, there have been inferences of distinct races in different parts of the United States and of the effects of maturity on resistance (Williams, 1981). Greenhalgh and Dickinson (1975) reported on the differential resistance of three crucifers to infection with $P$. parasitica on cotyledons and hypercots. However, these reports do not define a mature plant. Hoser-Krauze et al. (1987) considered a mature $B$. oleracea plant to be one that was in

Received for publication 8 Oct. 1992. Accepted for publication 4 Mar. 1993. The cost of publishing this paper was defrayed in part by the payment of page charges. Under postal regulations, this paper therefore must be hereby marked advertisement solely to indicate this fact. the four- to five-leaf stage. We thought 4-to 5week-old plants might not be mature in terms of resistance, and we tested older plants.

The objective of this study was to find out if broccoli plants resistant to the DM culture prevalent in one part of the United States would be resistant to the culture prevalent in other parts of the country. We also wanted to study the effect of plant maturity on resistance, since most studies had been on seedlings but resistance often was selected for in mature plants.

Inoculum was produced on susceptible seedlings, and spores were washed off cotyledons with water and sprayed with a hand sprayer onto seedlings and older test plants propagated by cuttings from field-grown plants. The plants were placed in a plastic bag for 24 $h$. Six days later, the plants were exposed to an atomized spray of water and returned to the plastic bag overnight. The following day, plants were removed from the bag and scored for disease. During disease testing, the plants were kept at $20 \pm 3 \mathrm{C}$. The seedlings were grown in trays (model 200; Speedling, Sun City, Fla.) with $5 \times 5 \times 7.5-\mathrm{cm}$ cubicles. For mature-plant studies, cuttings were removed from fieldgrown plants, dusted with the rooting compound Rootone (Miller Chemical and Fertilizer Corp., Hanover, Pa.), and placed in a mist chamber for 10 days. The rooted cuttings were transferred to 2-liter pots filled with Cornell mix (Boodley and Sheldrake, 1977).

Mature plants were selected from 49 lines of broccoli that were field resistant to DM in New York. We tested mature-plant cuttings from these 49 lines and found 40 to be resistant, 2 susceptible, and 7 intermediate. However, a seedling test of these same lines (eight seedlings per line per isolate source) with the same isolate source indicated 48 lines were susceptible and only one line was resistant to the same culture. This result clearly showed the influence of age on resistance and agreed with Williams (1981), who stated that seedling susceptibility does not mean that the plants will be equally susceptible when mature.

Using the same method described above, we screened plants of the original lines that Natti et al. (1967) found resistant as seedlings to race 1,2 , and 3 , respectively, against the major race present in New York in 1992. Plants resistant to races 1 and 3 were susceptible to race 2 when seedlings, but resistant when mature. This result is similar to the observation of Thomas et al. (1990), who found these plants susceptible to the prevalent race in South Carolina. Natti et al. (1967) tested the inbred line 68-M7-2, whose seedlings were resistant to the prevalent New York DM race, which they called race 2. As in 1967, this line's seedlings were resistant to the prevalent New York race in 1991; this also was the case in South Carolina (Thomas and Jourdain, 1990). The numbered lines referred to in the tables are breeding lines with resistance that traces back to 8777 or closely related lines. Line 8777 seedlings originally were resistant to race 2 in 1967, but seem to have lost this resistance in 1990. Since M-7-2 has not lost its resistance, 8777 seedlings may have lost their resistance during increase without selection.

Our results suggest an alternate rating system to the 1 to 5 scoring systems used by Natti et al. (1967), Williams (1981), Niu et al. (1983), and Thomas et al. (1990). In the Natti et al. (1967) scoring system, 1 = spots, necrotic flecks, streaks, but no sporulation; 2 = spots, necrotic flecks or streaks with sparse sporulation confined to necrotic tissue; and 3 to $5=$ systemic infection with sporulation in increasing degree. In the Williams (1981) system, $0=$ no symptoms; 1 = small necrotic flecks, no sporulation; 3 = necrotic flecks, one to few sporangiophores present; $5=$ necrotic lesions, sparse scattered sporulation usually confined to necrotic areas; 7 = necrotic lesions, sometimes with accompanying chlorosis, scattered, heavy to abundant sporulation in both chlorotic and necrotic areas; and $9=$ necrosis and some chlorosis evident, uniformly heavy sporulation over abaxial surface of leaf. Resistance classes based on disease indexes (DI) were calculated by Williams' formula: VR (very resistant $)=$ DI 0 to 3 ; MR (moderately resis$\operatorname{tant})=$ DI 3.1 to $5 ;$ LR (lowly resistant $)=$ DI 5.1 to $6 ; \mathrm{S}$ (susceptible) $=$ DI 6.1 to 7 ; and VS (very susceptible) $=$ DI 7.1 to 9 .

We used a disease score of 1 to 9: $1=$ no symptoms; 2 = slight necrotic flecking and no sporulation; 3 = necrotic flecking and no sporulation; $4=$ considerable necrotic flecking and tissue necrosis but no sporulation; $5=$ necrotic lesion and a few sporangiophores present confined to necrotic areas; 7 = necrotic lesions, sometimes with accompanying chlorosis, scattered, heavy to abundant sporulation in both chlorotic and necrotic areas, as defined by Thomas et al. (1990); and 9= necrosis and some chlorosis evident, along with uniformly heavy sporulation over the abaxial surface of 
the leaf. Resistance classes would be VR (very resistant) $=$ disease scores (DS) 1 to 2.5; MR $($ moderately resistant $)=$ DS 2.6 to $4.5 ;$ SR $($ slightly resistant $)=$ DS 4.6 to $6.5 ;$ MS $($ moderately susceptible) $=$ DS 6.6 to 8.0 ; and VS (very susceptible $)=$ DS 8.1 to 9 . We observed that there was a considerable range in degree of necrosis before initiation of sporulation; this scale gives a greater range in ratings for selecting for degrees of resistance without any sporulation; i.e., 1 to 4.5 .

If slight sporulation is observed, the plant cannot be considered as resistant as a plant with no sporulation, but it would be less susceptible than a plant with severe sporulation. In Williams' (1981) or Niu's et al. (1983) classification, as used by Thomas and Jourdain (1990), a score of 5 is considered resistant, but there can be slight sporulation. Our classification would call 1 to 4.5 resistant, with no sporulation but considerable necrosis in a 4.5 score, and still allow a degree of further selection in the resistant class for higher levels of resistance in classes 1 and 2 with no necrosis.

We found differences in resistance and susceptibility in mature plants (Table 1) and suggest that good mature-plant resistance may be selected for in some genotypes. 'Everest' is very resistant when mature, but susceptible as a seedling to the prevalent race. The transition from susceptibility to resistance with age is clearly evident in some lines (Table 2).

Natti et al. (1967) identified a single dominant gene for seedling resistance to each race,

Table 1. Mean and standard error (SE) for level of resistance (disease score) to downy mildew in mature broccoli plants.

\begin{tabular}{lc}
\hline Line & Disease score $\pm \mathrm{SE}$ \\
\hline Everest & $1.7 \pm 0.51$ \\
6742 & $3.3 \pm 2.16$ \\
6730 & $2.3 \pm 1.03$ \\
Sultan & $3.8 \pm 1.72$ \\
8777 & $3.3 \pm 1.21$ \\
Snow Crown & $5.2 \pm 1.53$ \\
Genesis & $6.3 \pm 1.50$ \\
6740 & $8.7 \pm 0.51$ \\
Acadia & $9.0 \pm 0.00$ \\
Packman & $9.0 \pm 0.00$ \\
\hline
\end{tabular}

${ }^{\mathrm{z}}$ Mean of eight or more plants per line.

'Disease score: 1 = highly resistant, 5 = sporulation evident, $9=$ very susceptible.

Table 2. Variation in level of resistance (disease score) to downy mildew race 2 with plant age in broccoli.

\begin{tabular}{lccccc}
\hline & \multicolumn{5}{c}{ Disease score \pm sE } \\
\cline { 2 - 6 } Line & \multicolumn{5}{c}{ Plant age at inoculation (days) } \\
\hline 6733 & $9.0 \pm 0^{y}$ & $7.5 \pm 0.76$ & $4.7 \pm 0.71$ & $6.3 \pm 2.45$ & $5.2 \pm 2.42$ \\
6742 & $9.0 \pm 0$ & $7.1 \pm 1.00$ & $4.9 \pm 1.00$ & $2.0 \pm 0.00$ & $4.7 \pm 1.75$ \\
6743 & $9.0 \pm 0$ & $8.4 \pm 0.79$ & $6.2 \pm 1.71$ & $5.0 \pm 2.76$ & $4.2 \pm 2.14$ \\
7055 & $9.0 \pm 0$ & $9.0 \pm 0.00$ & $8.9 \pm 0.35$ & $7.1 \pm 3.13$ & $6.8 \pm 2.16$ \\
7804 & $9.0 \pm 0$ & $8.9 \pm 0.38$ & $8.7 \pm 0.71$ & $5.2 \pm 1.00$ & $6.3 \pm 0.51$ \\
8775 & $9.0 \pm 0$ & $7.3 \pm 0.79$ & $8.9 \pm 0.35$ & $6.7 \pm 1.50$ & $6.6 \pm 2.79$ \\
8777 & $9.0 \pm 0$ & $7.9 \pm 0.85$ & $5.1 \pm 1.00$ & $3.2 \pm 2.14$ & $7.7 \pm 0.81$ \\
Sultan & $9.0 \pm 0$ & $6.4 \pm 0.79$ & $4.3 \pm 0.48$ & $4.3 \pm 2.58$ & $4.0 \pm 1.55$ \\
Everest & $9.0 \pm 0$ & $5.0 \pm 2.16$ & $3.5 \pm 1.60$ & $3.2 \pm 1.50$ & $1.7 \pm 0.51$ \\
Mean & 9.0 & 7.53 & 6.23 & 5.54 & 4.70 \\
\hline
\end{tabular}

${ }^{\mathrm{z}}$ Disease score: 1 = highly resistant, $5=$ sporulation evident, $9=$ very susceptible.

'Eight plants were tested per line and age group. but also indicated that some plants classified as resistant were only moderately resistant, as we observed for 8777 . There is a considerable range in resistance or susceptibility in mature plants (as evaluated by our disease scores) (Table 1), thus permitting selection for lines with high levels of resistance. When screening large plants compared to seedlings, it is easier to discern intermediate levels of resistance. However, it is much easier to test large seedling populations. Our observations confirm those of Natti et al. (1967) that resistance is dominant and that there maybe a single dominant gene. However, the continuous range in degree of resistance indicates the action of modifying genes.

To determine the influence of isolate source on resistance, isolates from California, Washington, New York, and South Carolina were used to inoculate cuttings from 74 mature plants. Of the 74 plants, the scores for 46 varied by 0 or 1 among isolate sources. The other 38 clones had, in almost every case, one plant with a disease score 22 units outside the classification range. Sixteen lines were uniformly very resistant (1 to 2), and six had one score $>1$ or 2 . Six lines scored uniformly low resistance (5 to 6) and nine showed some variation. Fourteen lines were uniformly intermediately susceptible (6 to 8) and eleven had some variation, ten lines were uniformly very susceptible (8 to 9 ) and four showed some variation, The disease scores (from plants selected as examples) of each cutting inoculated with spores from one of the four geographical regions show the uniformity of response to the various isolate sources (Table 3 ). The correlations between the scores from the four isolates were all highly significant at $P \leq 0.01$ (Table 4). This result indicates that there are no real differences in isolate sources among the four regions of the United States and that resistance developed in any one region likely would stand up in all the others.

The study reported here suggests that breeders can screen physiologically mature plants (seven or more leaves), save those with disease scores of 1,2 , or possibly 3 , thus advancing those with distinct and high levels of resistance to DM. Such resistance may not be preserved by seedling screening alone. If juve-

Table 3. Downy mildew disease scores after inoculating broccoli with isolates from four locations. Examples from 74 lines were selected to show uniformity and variability among and within lines. $^{z}$

\begin{tabular}{lllll}
\hline & \multicolumn{4}{c}{ Disease score $^{y}$} \\
\cline { 2 - 5 } Line & \multicolumn{4}{c}{ Inoculum source } \\
\cline { 2 - 5 } & S.C. & Calif. & Wash. & N.Y. \\
\hline $6873-1$ & 5 & 6 & 5 & 5 \\
$6874-1$ & 7 & 8 & 6 & 8 \\
$6884-2$ & 6 & 3 & 7 & 8 \\
$6886-1$ & 9 & 9 & 9 & 9 \\
$6892-1$ & 2 & 2 & 3 & 1 \\
$6903-1$ & 1 & 1 & 1 & 1 \\
$6911-1$ & 1 & 1 & 1 & 1 \\
$6913-1$ & 4 & 9 & 6 & 8 \\
$6914-1$ & 8 & 9 & 7 & 8 \\
$6740-1$ & 6 & 3 & 6 & 2 \\
Mean & 4.93 & 5.36 & 5.20 & 5.45 \\
\hline
\end{tabular}

${ }^{2}$ One cutting from each line was tested with each inoculum source.

'Disease score: 1 = highly resistant, 5 = sporulation evident, $9=$ very susceptible.

'S.C. = South Carolina; Calif. = California; Wash. = Washington; N.Y. = New York.

Table 4. Correlation of downy mildew disease scores on broccoli among various downy mildew isolates from four sources.

\begin{tabular}{lccc}
\hline & \multicolumn{3}{c}{ Correlation coefficient } \\
\cline { 2 - 4 } & \multicolumn{3}{c}{ Isolate source } \\
\cline { 2 - 4 } Isolate source & S.C. & Calif. & Wash. \\
\hline Calif. & $0.648^{* *}$ & & \\
Wash. & $0.736^{* *}$ & $0.483^{* *}$ & \\
N.Y. & $0.596^{* *}$ & $0.670^{* *}$ & $0.872^{* *}$
\end{tabular}

${ }^{7}$ S.C. = South Carolina; Calif. = California; Wash. = Washington; N.Y. = New York.

${ }^{* *}$ Significant at $P \leq 0001$.

nile and mature-plant resistance are needed, then screening seedlings (cotyledon or plants with one or two true leaves) and mature plants will be necessary.

\section{Literature Cited}

Boodley, J.W. and R. Sheldrake, Jr. 1977. Cornell Peat-Lite mixes for commercial plant growing. Cornell Univ. Plant Sci. Info. Bul. 43.

Greenhalgh, J.R. and C.H. Dickinson. 1975. Differential reaction of 3 crucifers to infection by Peronospora parasitica (pers. ex Fr) Fr. Phytopathol. Z. 84:131-141.

Hoser-Krauze, J., E. Lakowska-Ryk, and J. Antosik. 1987. The inheritance of broccoli (Brassica oleracea L. var. botrytis) leaf resistance to downy mildew -Peronspora parasitica (pers.) ex. Fr. Genetica Polonica 28:377-380.

Natti, J.J., M.H. Dickson, and J.D. Atkin. 1967. Resistance of Brassica oleracea varieties to downy mildew. Phytopathology 57:144-157.

Niu, X-K, N. Lueng, and P.H, Williams. 1983. Sources and nature of resistance to downy mildew and turnip mosaic in Chinese cabbage. J. Amer. Soc. Hort. Sci. 108:775-778.

Thomas, C.E. and L. Jourdain. 1990. Evacuation of broccoli and cauliflower germplasm for resistance to race 2 of Peronospora parasitica. HortScience 25:1429-1431.

Williams, P.H. 1981. Crucifer genetics resource book. Dept. of Plant Pathology, Univ. of Wisconsin, Madison. 